\title{
Effect of different palatal vault shapes on the dimensional stability of glass fiber- reinforced heat-polymerized acrylic resin denture base material
}

\author{
Mehmet Dalkiz \\ Demet Arslan² \\ Ali Riza Tuncdemir ${ }^{1}$ \\ M.Selim Bilgin ${ }^{3}$ \\ Halil Aykul ${ }^{4}$
}

\section{ABSTRACT}

Objective: The aim of this study was to determine the effect of different palatal vault shapes on the dimensional stability of a glass fiber reinforced heat polymerized acrylic resin denture base material.

Methods: Three edentulous maxilla with shallow, deep and medium shaped palatal vaults were selected and elastomeric impressions were obtained. A maxillary cast with four reference points ( $A$, $B, C$, and D) was prepared to serve as control. Point (A) was marked in the anterior midline of the edentulous ridge in the incisive papillary region, points $(B)$ and $(C)$ were marked in the right and left posterior midlines of the edentulous ridge in the second molar regions, and point (D) was marked in the posterior palatal midline near the fovea palatina media (Figure 2). To determine linear dimensional changes, distances between four reference points $(A-B, A-C, A-D$ and $B-C$ ) were initially measured with a metal gauge accurate within $0.1 \mathrm{~mm}$ under a binocular stereo light microscope and data $(\mathrm{mm})$ were recorded.

Results:No significant difference of interfacial distance was found in sagittal and frontal sections measured $24 \mathrm{~h}$ after polymerization and after 30 days of water storage in any of experimental groups $(P>$.05). Significant difference of linear dimension were found in all experimental groups $(P<.01)$ between measurements made $24 \mathrm{~h}$ after polymerization of specimens and 30 days after water storage.

Conclusion: Palatal vault shape and fiber impregnation into the acrylic resin bases did not affect the magnitude of interfacial gaps between the bases and the stone cast surfaces. (Eur J Dent 2012;6:70-78)

Key Words: Acrylic resin denture base, denture reinforcing fiber, polymerization, dimensional stability, palatal vault shape.

1 Department of Prosthetic Dentistry, Faculty of Dentistry, Mustafa Kemal University, Antakya, TURKIYE.

2 Department of Prosthetic Dentistry, Dental Sciences Center, Gulhane Military Medical Academy, Ankara, TURKIYE.

3 Department of Prosthetic Dentistry, Faculty of Dentistry, Ishik University, Erbil, IRAQ.
4 Department of Mechanics, Engineering Faculty, Hitit University, Corum, TURKIYE.

- Corresponding author: Dr. Ali Riza Tuncdemir Address: Mustafa Kemal University, Dentistry Faculty, Hatay, TURKIYE.

Phone: +900326 2291000 Fax: +90 03262455654 E-Mail: alirizatuncdemiragmail.com 


\section{INTRODUCTION}

The number of denture wearers is increasing as the number of elderly people is continually growing and polymethyl methacrylate (PMMA) is still the most frequently used material in denture base fabrication. ${ }^{1}$ Despite of its satisfactory aesthetic properties, ease of processing and accurate fit, PMMA incurs dimensional changes during processing and use. ${ }^{2-5}$ Thickness of the denture, thermal expansion and contraction of the acrylic resin and the gypsum, structural variations between polymer and monomer systems, polymer/ monomer ratios, packing pressure, polymerization shrinkage, investments, separators, internal stresses, water sorption and desorption and water content of the gypsum were reported as some variables influencing the dimensional stability of an acrylic resin denture..$^{2-10}$ In recent years, various types of reinforcing fibers have been introduced and used to enhance the physical and mechanical properties of acrylic resin denture bases and to increase their clinical performance. ${ }^{11-22}$ Effects of the type ${ }^{11-16}$, the form ${ }^{17,18}$, the length ${ }^{19,20}$ and the concentration ${ }^{20}$ of reinforcing fibers, as well as the type of fiber-reinforced acrylic resin denture base material ${ }^{21}$ on the dimensional stability were investigated in previous studies. However, a study demonstrating the relation between the palatal vault shape and the dimensional stability of fiber reinforced denture base materials was not conducted up to now. The purpose of this in vitro study was to determine the effect of different palatal vault shapes on the dimensional stability of a glass fiber reinforced heat polymerized acrylic resin denture base material.

\section{MATERIALS AND METHODS}

Three edentulous maxilla with shallow, deep and normal shaped palatal vaults were selected and impressions were obtained with an elastomeric impression material (Zetaplus; Zhermack Spa, Badia Polesine, Rovigo, Italy) (Figure 1). Each impression was cast and duplicated 30 times with Type IV dental stone (Begostone; Bego, Bremer GW Herbst $\mathrm{GmbH}$ \& Co, Bremen, Germanyl and totally 90 definitive casts were obtained. Heat polymerized acrylic resin denture bases (QC-20, Dentsply Int Inc, Waybridge, Surrey, UK) were fabricated onto each definitive cast according to the recommendations of the manufacturer and pre- pared specimens were assigned to 3 main experimental groups (Table 1) representing three different vault shapes: Group SHAL ROUNDED Group U (U-shaped) and Group V (V-shaped) ( $n=30)$. Each main group consisted of 3 subgroups $(n=10)$ representing the type of fiber used; subgroups including wowen type fibers (Groups ROUNDED/WOF, U/ WOF, V/WOF), subgroups including roving type fibers (Groups ROUNDED/ROF, U/ROF, and V/ROF), and finally subgroups which did not include any fiber (Groups ROUNDED/NOF, U/NOF, V/NOF).

An epoxy resin maxillary cast (Polycast Industries, Bay Shore, NY) with four reference points (A, $B, C$, and D) was prepared to serve as control for metal gauge measurements. Point (A) was marked in the anterior midline of the edentulous ridge in the incisive papillary region, points (B) and (C) were marked in the right and left posterior midlines of the edentulous ridge in the second molar regions, and point (D) was marked in the posterior palatal midline near the fovea palatina media (Figure 2). To determine linear dimensional changes, distances between four reference points $(A-B, A-C$, $A-D$ and $B-C$ ) were initially measured with a metal gauge accurate within $0.1 \mathrm{~mm}$ (Hu-Friedy, Chicago, IL) under a binocular stereo light microscope (Baytronix, B000A7XBSQ, Silver Spring, MD) with 20x magnification and data $(\mathrm{mm})$ were recorded.

Preparation of specimens without fiber

For specimen preparation of groups ROUNDED/NOF, U/NOF and V/NOF, 2 sheets of modeling wax were spread onto the casts as to completely cover the surfaces. A small wax extension was adapted at the posterior border of each cast to provide shrinkage compensation during the polymerization process. Cast specimens covered with wax patterns were flasked with Type II dental stone (Moldano, Bayer, Germany) and were allowed to set for 45 minutes. The flasks were then placed in boiling water for five minutes to soften the waxes. The flasks were then opened and the wax elimination was performed. Cast surfaces were thoroughly cleansed with liquid soap and rinsed with boiling water (Boil Out Unit, EWL 5522, Kavo EWL, Germany). Two coats of separator (DVA Acrylic and Plaster Separator, DVA Inc, Corona, CA) were applied on cast surfaces and allowed to dry. Heat polymerized acrylic resin denture base material (QC-20, Dentsply Int Inc, Waybridge, Sur- 
rey, UKJ was prepared according to the recommendations of the manufacturer. The prepared acrylic resin dough was allowed to set for $20 \mathrm{~min}$ and was packed into the flasks with finger pressure. Following trial closures for removing excess resin, the flasks were closed, pressed in a hydraulic pressing apparatus (KaVo Elektrotechnisches, D-7970, Werk Germanyl under a pressure of 100 bars and heated for 30 minutes at $75^{\circ} \mathrm{C}$ and an additional 30 minutes at $100^{\circ} \mathrm{C}$.

Preparation of specimens reinforced with woven type glass fibers

For specimen preparation of groups ROUNDED/WOF, U/WOF and V/WOF, woven type glass fi- bers (Vetrolex RC, 14-800-P109, Ciba Composites, Birmingham, UK) were shaped with a lancet as to provide $2 \mathrm{~mm}$ shorter borders than the boundaries of acrylic resin bases. Heat polymerized acrylic resin denture base material (QC-20, Dentsply Int Inc, Waybridge, Surrey, UK) was prepared according to the recommendations of the manufacturer. To provide accurate penetration and integration, shaped glass fibers were dipped in monomer for 15 min before being introduced into the acrylic resin bulk. A thin layer of acrylic resin dough was first placed on the cast surfaces inside the flask and prepared woven fibers were placed on the initially placed acrylic layer. A second thin layer of acrylic resin dough was placed on the woven fiber
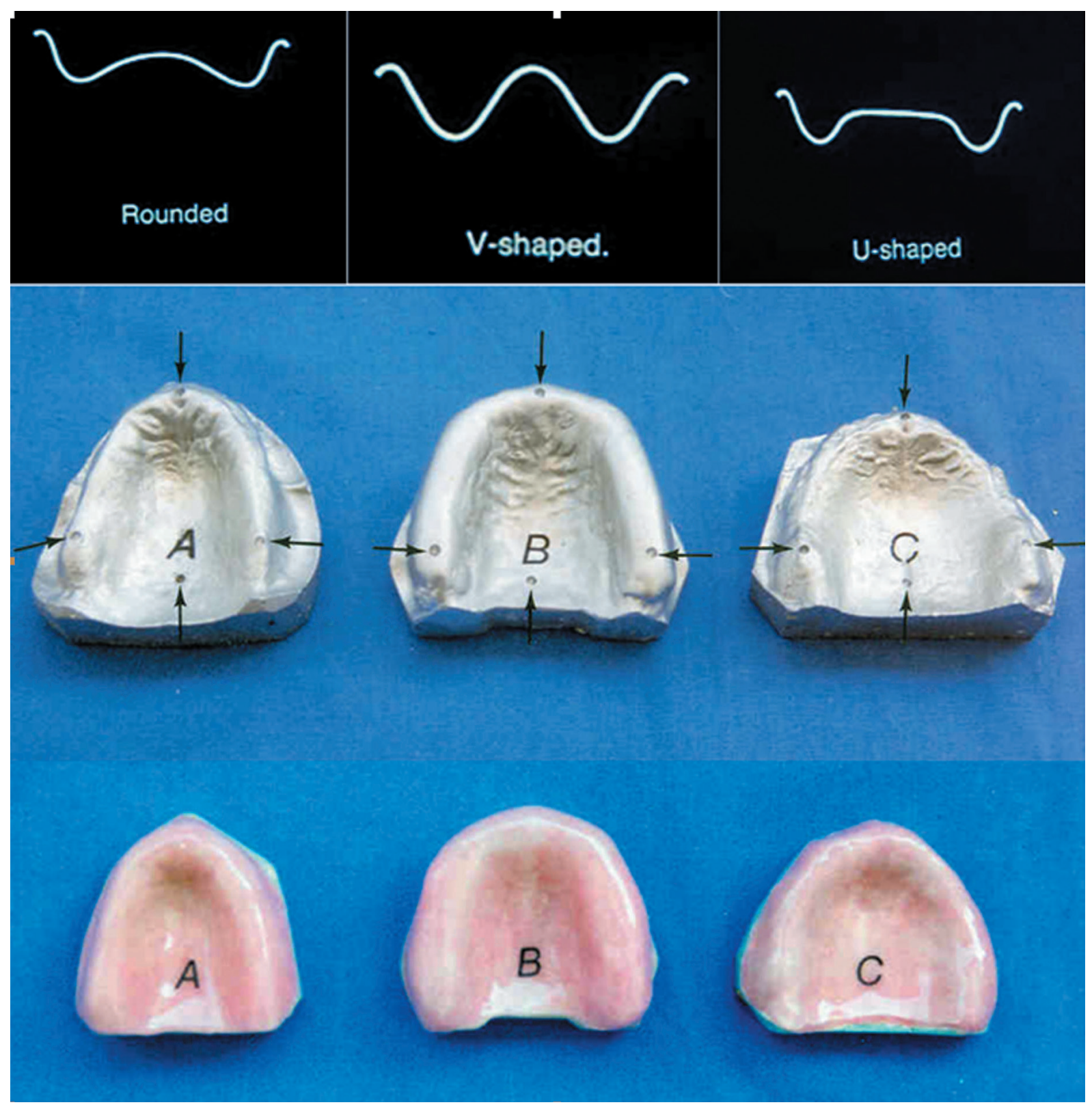

Figure 1. Definitive casts obtained from different palatal vault rounded (V: A, Uium: B, rounded: C), and fiber reinforced acrylic resin bases. The arrows demonstrate marked reference points $(A, B, C, D)$ 
surface with finger pressure. Following trial closures for removing excess resin, the flasks were closed, pressed and heated in a previously described manner.

Preparation of specimens reinforced with roving type glass fibers

For specimen preparation of groups ROUNDED/ROF, U/ROF and V/ROF containing roving type glass fibers (Vetrolex RC, 14-800-P-109, Ciba Composites, Birmingham, UK), fibers were cut 0.5 $\mathrm{mm}$ in length with a lancet. The fiber content was calculated from the initial weight of the specimens to an accuracy of $0.01 \mathrm{mg}$ using a digital device (Mettler, Zurich, Switzerland). ${ }^{15}$ The weight of fibers was determined with a ratio of $10 \%$ by weight of the polymer matrix and impregnated with appropriate matching to polymer/monomer mixture. Prepared roving glass fibers were directly mixed with the polymer. The acrylic resin dough was prepared in a previously described manner and packed into the flask with finger pressure. Following trial closures for removing excess resin, the flasks were closed, pressed and heated in a previously described manner.

\section{Measurement of interfacial distances}

Reference points $A, B, C$ and $D$ were marked as previously described onto each acrylic resin base specimen and distances between these reference

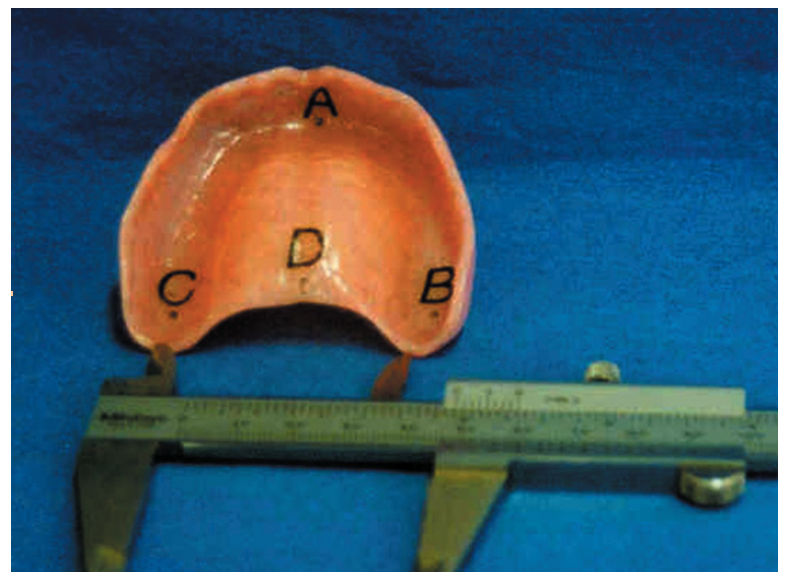

Figure 2. Linear distances between marked reference points A, B, C and D measured with a metal gauge.

Table 1. Main experimental groups representing palatal vault shapes and subgroups representing the fiber types used.

\begin{tabular}{lcccc}
\hline (Vault shape) & No fiber (NOF) & Woven (WOF) & Roving (ROF) & Total \\
\hline ROUND & ROUND/NOF & ROUND/WOF & ROUND/ROF & 30 \\
U-SHAPED & U/NOF & U/WOF & U/ROF & 30 \\
V-SHAPED & V/NOF & V/WOF & V/ROF & 30 \\
\hline
\end{tabular}

Subgroups $(n=10)$

January 2012 - Vol.6 points were measured to evaluate the probable linear dimensional change (Figure 2). Measurements were performed at these reference points using a metal gauge and a binocular stereo light microscope, under 20x magnification, 24 hours after the polymerization process. A second measurement was performed after the storage of the specimens in water for 30 days at room temperature. Obtained data were recorded in millimeters.

To determine the adaptation or the interfacial gap between acrylic resin bases and the stone casts after water storage, the specimens were sagittaly sectioned with a precision rotary microtome (Slee Cut, 6062, NIC Medikal, Izmir, Turkey) in an anteroposterior direction along the imaginary line passing between reference points $A$ and $D$ (Figure 2 and 3 ). A second frontal cross section - perpendicular to the sagittal section - was performed along a path passing between right and left first molar regions (Figure 2 and 3). To measure the interfacial distance between the cast surface and the inner surface of acrylic resin bases, seven reference points were marked along the resin base/stone cast interface in sagittal sections and five reference points were marked at frontal cross sections (Figure 3). Measurements were performed at these reference points as previously described, 24 hours after the polymerization process. A second measurement was performed after the storage of the specimens in water for 30 days

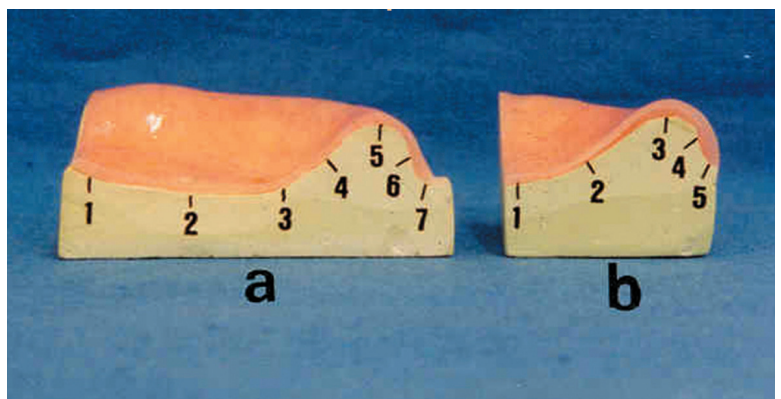

Figure 3. Sagittal (a) and frontal (b) sections of test specimens and interfacial reference points. 
at room temperature. Obtained data were recorded in millimeters.

\section{Statistical analysis}

A statistical software program (SPSSFW, 9.0; SPSS Inc, Chicago, III) was used for statistical analyses. Data obtained at the first and second distance measurements were compared with Kruskal-Wallis analysis of variance test. Signifi- cant differences between groups were compared using Mann-Whitney $U$ test with Bonferroni correction. A 95\% confidence level was used.

\section{RESULTS}

Mean, standard deviation and $P$ values of data $(\mathrm{mm})$ obtained from 7 marked regions in sagittal sections of test specimens $24 \mathrm{~h}$ after polymerization and after 30 days of water storage are pre-

Table 2. Mean, standard deviation and P values of linear measurement data $(\mathrm{mm})$ obtained from resin/stone cast interfaces in 7 marked regions in sagittal sections of test specimens, $24 \mathrm{~h}$ after polymerization and after 30 days of water storage (confidence level: $95 \%, \mathrm{P}<.05$ ).

\begin{tabular}{|c|c|c|c|c|c|c|c|c|c|c|c|c|c|}
\hline \multicolumn{2}{|c|}{ Sagittal sections } & \multicolumn{4}{|c|}{ NOF } & \multicolumn{4}{|c|}{ WOF } & \multicolumn{4}{|c|}{ ROF } \\
\hline Region & $\begin{array}{c}\text { Time of } \\
\text { measurement }\end{array}$ & Groups & Mean & St. Dev. & $P$ & Groups & Mean & St. Dev. & $P$ & Groups & Mean & St. Dev. & $P$ \\
\hline \multirow{6}{*}{1} & \multirow{3}{*}{$\begin{array}{c}24 \mathrm{~h} \text { (after } \\
\text { polymerization) }\end{array}$} & u & 0.183 & 0.090 & \multirow{3}{*}{$>0.05$} & u & 0.131 & 0.164 & \multirow{3}{*}{$>0.05$} & U & 0.153 & 0.100 & \multirow{3}{*}{$>0.05$} \\
\hline & & v & 0.196 & 0.112 & & v & 0.087 & 0.08 & & V & 0.144 & 0.064 & \\
\hline & & ROUNDED & 0.158 & 0.083 & & ROUNDED & 0.100 & 0.070 & & ROUNDED & 0.129 & 0.070 & \\
\hline & \multirow{3}{*}{$\begin{array}{c}30 \text { days (water } \\
\text { storage) }\end{array}$} & U & 0.144 & 0.143 & \multirow{3}{*}{$>0.05$} & U & 0.136 & 0.151 & \multirow{3}{*}{$>0.05$} & U & 0.136 & 0.109 & \multirow{3}{*}{$>0.05$} \\
\hline & & v & 0.113 & 0.073 & & v & 0.109 & 0.098 & & V & 0.097 & 0.077 & \\
\hline & & ROUNDED & 0.111 & 0.084 & & ROUNDED & 0.050 & 0.050 & & ROUNDED & 0.100 & 0.060 & \\
\hline \multirow{6}{*}{2} & \multirow{3}{*}{24 h (a.p.) } & $U$ & 0.153 & 0.077 & \multirow{3}{*}{$>0.05$} & U & 0.079 & 0.140 & \multirow{3}{*}{$>0.05$} & $U$ & 0.118 & 0.080 & \multirow{3}{*}{$>0.05$} \\
\hline & & v & 0.118 & 0.067 & & v & 0.045 & 0.019 & & v & 0.128 & 0.077 & \\
\hline & & ROUNDED & 0.138 & 0.095 & & ROUNDED & 0.070 & 0.060 & & ROUNDED & 0.106 & 0.144 & \\
\hline & \multirow{3}{*}{30 days (w.s.) } & $U$ & 0.133 & 0.034 & \multirow{3}{*}{$>0.05$} & U & 0.083 & 0.116 & \multirow{3}{*}{$>0.05$} & U & 0.121 & 0.121 & \\
\hline & & v & 0.060 & 0.055 & & v & 0.048 & 0.047 & & v & 0.067 & 0.064 & $>0.05$ \\
\hline & & ROUNDED & 0.088 & 0.066 & & ROUNDED & 0.040 & 0.030 & & ROUNDED & 0.060 & 0.060 & \\
\hline & & U & 0.116 & 0.058 & & U & 0.036 & 0.088 & & u & 0.080 & 0.070 & \\
\hline & 24 h (a.p.) & v & 0.093 & 0.058 & $>0.05$ & v & 0.085 & 0.147 & $>0.05$ & v & 0.095 & 0.093 & $>0.05$ \\
\hline 3 & & ROUNDED & 0.141 & 0.140 & & ROUNDED & 0.050 & 0.060 & & ROUNDED & 0.070 & 0.100 & \\
\hline 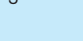 & & U & 0.082 & 0.034 & & U & 0.044 & 0.102 & & u & 0.100 & 0.100 & \\
\hline & 30 days (w.s.) & v & 0.055 & 0.032 & $>0.05$ & v & 0.037 & 0.022 & $>0.05$ & v & 0.068 & 0.083 & $>0.05$ \\
\hline & & ROUNDED & 0.080 & 0.050 & & ROUNDED & 0.040 & 0.080 & & ROUNDED & 0.040 & 0.050 & \\
\hline & & U & 0.097 & 0.092 & & U & 0.021 & 0.040 & & U & 0.020 & 0.020 & \\
\hline & 24 h (a.p.) & v & 0.053 & 0.035 & $>0.05$ & DEP & 0.033 & 0.049 & $>0.05$ & v & 0.073 & 0.097 & $>0.05$ \\
\hline 4 & & ROUNDED & 0.055 & 0.037 & & ROUNDED & 0.010 & 0.020 & & ROUNDED & 0.020 & 0.040 & \\
\hline 4 & & u & 0.082 & 0.083 & & u & 0.039 & 0.082 & & U & 0.030 & 0.060 & \\
\hline & 30 days (w.s.) & v & 0.027 & 0.020 & $>0.05$ & v & 0.008 & 0.014 & $>0.05$ & v & 0.050 & 0.102 & $>0.05$ \\
\hline & & ROUNDED & 0.042 & 0.033 & & ROUNDED & 0.020 & 0.050 & & ROUNDED & 0.020 & 0.020 & \\
\hline & & U & 0.072 & 0.113 & & U & 0.013 & 0.021 & & U & 0.010 & 0.020 & \\
\hline & 24 h (a.p.) & v & 0.017 & 0.036 & $>0.05$ & v & 0.013 & 0.023 & $>0.05$ & v & 0.028 & 0.053 & $>0.05$ \\
\hline 5 & & ROUNDED & 0.018 & 0.032 & & ROUNDED & 0.010 & 0.020 & & ROUNDED & 0.010 & 0.020 & \\
\hline 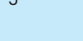 & & u & 0.012 & 0.021 & & u & 0.003 & 0.006 & & u & 0.002 & 0.005 & \\
\hline & 30 days (w.s.) & v & 0.005 & 0.008 & $>0.05$ & v & 0.010 & 0.022 & $>0.05$ & v & 0.010 & 0.016 & $>0.05$ \\
\hline & & ROUNDED & 0.013 & 0.019 & & ROUNDED & 0.003 & 0.007 & & ROUNDED & 0.007 & 0.010 & \\
\hline & & u & 0.047 & 0.078 & & U & 0.003 & 0.006 & & u & 0.020 & 0.060 & \\
\hline & 24 h (a.p.) & v & 0.018 & 0.036 & $>0.05$ & v & 0.006 & 0.016 & $>0.05$ & v & 0.027 & 0.051 & $>0.05$ \\
\hline t & & ROUNDED & 0.027 & 0.031 & & ROUNDED & 0.020 & 0.020 & & ROUNDED & 0.010 & 0.010 & \\
\hline 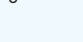 & & u & 0.013 & 0.028 & & u & 0.001 & 0.005 & & u & 0.002 & 0.005 & \\
\hline & 30 days (w.s.) & v & 0.008 & 0.014 & $>0.05$ & v & 0.008 & 0.021 & $>0.05$ & v & 0.017 & 0.027 & $>0.05$ \\
\hline & & ROUNDED & 0.015 & 0.017 & & ROUNDED & 0.005 & 0.008 & & ROUNDED & 0.005 & 0.008 & \\
\hline & & U & 0.077 & 0.078 & & u & 0.011 & 0.022 & & U & 0.050 & 0.070 & \\
\hline & 24 h (a.p.) & v & 0.023 & 0.034 & $>0.05$ & v & 0.038 & 0.054 & $>0.05$ & v & 0.030 & 0.026 & $>0.05$ \\
\hline 1 & & ROUNDED & 0.033 & 0.018 & & ROUNDED & 0.030 & 0.030 & & ROUNDED & 0.010 & 0.020 & \\
\hline & 30 days (w.s.) & U & 0.028 & 0.029 & $>0.05$ & U & 0.004 & 0.008 & $>0.05$ & U & 0.010 & 0.009 & $>0.05$ \\
\hline
\end{tabular}


sented at Table 2. Mean, standard deviation and $P$ values of data $(\mathrm{mm})$ obtained from 5 marked regions in frontal sections of test specimens 24 $\mathrm{h}$ after polymerization and after 30 days of water storage are presented at Table 3. Mean, standard deviation and $P$ values of data $(\mathrm{mm})$ obtained from linear measurements of distances between 4 references points $(A, B, C$, and $D)$ in medium palatal vault shape groups $24 \mathrm{~h}$ after polymerization and after 30 days of water storage are presented at Table 4.

Mean, standard deviation and $P$ values of data $(\mathrm{mm})$ obtained from linear measurements of distances between 4 references points in deep palatal vault shape groups $24 \mathrm{~h}$ after polymerization and after 30 days of water storage are presented at Table 5. Mean, standard deviation and $P$ values of data $(\mathrm{mm})$ obtained from linear measurements of distances between 4 references points in shallow palatal vault shape groups $24 \mathrm{~h}$ after polymerization and after 30 days of water storage are presented at Table 6.

According to data of statistical analyses, the results of the present study are as follows.

Not any significant distance difference was found at acrylic resin/stone cast interfaces measured at 7 marked regions in sagittal sections, 24 hours after polymerization and after 30 days of water storage, in any of the experimental groups (P>.05) (Table 2).

Not any significant linear dimensional difference was found in acrylic resin/stone cast interfacial distances measured at 5 marked regions in frontal sections, 24 hours after polymerization and

Table 3. Mean, standard deviation and $P$ values of linear measurement data $(\mathrm{mm})$ obtained from resin/stone cast interfaces in 5 marked regions in frontal sections of test specimens, $24 \mathrm{~h}$ after polymerization and after 30 days of water storage $(P<.05)$.

\begin{tabular}{|c|c|c|c|c|c|c|c|c|c|c|c|c|c|}
\hline \multicolumn{2}{|c|}{ Frontal sections } & \multicolumn{4}{|c|}{ NOF } & \multicolumn{4}{|c|}{ WOF } & \multicolumn{4}{|c|}{ ROF } \\
\hline Region & $\begin{array}{l}\text { Time of linear } \\
\text { measurement }\end{array}$ & Groups & Mean & St. Dev. & $\mathrm{P}$ & Groups & Mean & St. Dev. & $\mathrm{P}$ & Groups & Mean & St. Dev. & $P$ \\
\hline \multirow{6}{*}{1} & & U & 0.139 & 0.072 & & U & 0.051 & 0.067 & & U & 0.126 & 0.146 & \\
\hline & $24 \mathrm{~h}$ (a.p.) & v & 0.149 & 0.067 & $>0.05$ & v & 0.060 & 0.030 & $>0.05$ & V & 0.104 & 0.067 & $>0.05$ \\
\hline & & ROUNDED & 0.123 & 0.060 & & ROUNDED & 0.070 & 0.60 & & ROUNDED & 0.114 & 0.101 & \\
\hline & & U & 0.093 & 0.060 & & U & 0.049 & 0.052 & & U & 0.100 & 0.070 & \\
\hline & 30 days (w.s.) & v & 0.114 & 0.080 & $>0.05$ & $v$ & 0.032 & 0.025 & $>0.05$ & v & 0.098 & 0.072 & $>0.05$ \\
\hline & & ROUNDED & 0.100 & 0.70 & & ROUNDED & 0.060 & 0.050 & & ROUNDED & 0.060 & 0.060 & \\
\hline \multirow{6}{*}{2} & & U & 0.092 & 0.079 & & $u$ & 0.039 & 0.052 & & U & 0.060 & 0.060 & \\
\hline & $24 \mathrm{~h}$ (a.p.) & v & 0.070 & 0.034 & $>0.05$ & v & 0.020 & 0.020 & $>0.05$ & v & 0.065 & 0.049 & $>0.05$ \\
\hline & & ROUNDED & 0.082 & 0.056 & & ROUNDED & 0.030 & 0.040 & & ROUNDED & 0.060 & 0.060 & \\
\hline & & U & 0.062 & 0.051 & & u & 0.026 & 0.040 & & U & 0.030 & 0.040 & \\
\hline & 30 days (w.s.) & v & 0.062 & 0.049 & $>0.05$ & v & 0.010 & 0.014 & $>0.05$ & v & 0.058 & 0.052 & $>0.05$ \\
\hline & & ROUNDED & 0.077 & 0.049 & & ROUNDED & 0.010 & 0.020 & & ROUNDED & 0.060 & 0.050 & \\
\hline \multirow{6}{*}{3} & & U & 0.032 & 0.054 & & U & 0.023 & 0.029 & & U & 0.008 & 0.010 & \\
\hline & 24 h (a.p.) & v & 0.016 & 0.014 & $>0.05$ & v & 0.008 & 0.014 & $>0.05$ & v & 0.015 & 0.023 & $>0.05$ \\
\hline & & ROUNDED & 0.015 & 0.023 & & ROUNDED & 0.008 & 0.010 & & ROUNDED & 0.020 & 0.030 & \\
\hline & & U & 0.013 & 0.027 & & U & 0.006 & 0.011 & & U & 0.010 & 0.020 & \\
\hline & 30 days (w.s.) & v & 0.011 & 0.013 & $>0.05$ & v & 0.05 & 0.011 & $>0.05$ & v & 0.006 & 0.012 & $>0.05$ \\
\hline & & ROUNDED & 0.008 & 0.014 & & ROUNDED & 0.007 & 0.010 & & ROUNDED & 0.010 & 0.020 & \\
\hline \multirow{6}{*}{4} & & U & 0.010 & 0.021 & & U & 0.013 & 0.021 & & U & 0.030 & 0.080 & \\
\hline & $24 \mathrm{~h}$ (a.p.) & V & 0.016 & 0.021 & $>0.05$ & v & 0.012 & 0.014 & $>0.05$ & v & 0.022 & 0.026 & $>0.05$ \\
\hline & & ROUNDED & 0.012 & 0.016 & & ROUNDED & 0.010 & 0.020 & & ROUNDED & 0.010 & 0.020 & \\
\hline & & U & 0.008 & 0.012 & & u & 0.009 & 0.014 & & U & 0.008 & 0.010 & \\
\hline & 30 days (w.s.) & V & 0.013 & 0.015 & $>0.05$ & v & 0.005 & 0.008 & $>0.05$ & v & 0.008 & 0.012 & $>0.05$ \\
\hline & & ROUNDED & 0.006 & 0.012 & & ROUNDED & 0.005 & 0.010 & & ROUNDED & 0.005 & 0.008 & \\
\hline \multirow{6}{*}{5} & & U & 0.027 & 0.025 & & U & 0.029 & 0.023 & & U & 0.030 & 0.020 & \\
\hline & 24 h (a.p.) & v & 0.035 & 0.019 & $>0.05$ & V & 0.022 & 0.022 & $>0.05$ & V & 0.047 & 0.035 & $>0.05$ \\
\hline & & ROUNDED & 0.027 & 0.020 & & ROUNDED & 0.020 & 0.020 & & ROUNDED & 0.020 & 0.020 & \\
\hline & & U & 0.020 & 0.017 & & U & 0.029 & 0.020 & & U & 0.020 & 0.020 & \\
\hline & 30 days (w.s.) & v & 0.034 & 0.021 & $>0.05$ & v & 0.012 & 0.014 & $>0.05$ & v & 0.040 & 0.062 & $>0.05$ \\
\hline & & ROUNDED & 0.015 & 0.015 & & ROUNDED & 0.010 & 0.010 & & ROUNDED & 0.010 & 0.009 & \\
\hline
\end{tabular}


after 30 days of water storage, in any of the experimental groups (P>.05) (Table 3).

In U-SHAPED groups; significant differences were found between linear measurements performed $24 \mathrm{~h}$ after polymerization and after 30 days of water storage in all groups (U/NOF, U/WOF, U/ ROF) $P<.01$ (Table 4).

In V-SHAPED groups; significant differences were found between linear measurements per- formed $24 \mathrm{~h}$ after polymerization and after 30 days of water storage in all groups (V/NOF, V/WOF, V/ ROF) ( $P<.01$ ) (Table 5).

In ROUNDED groups; significant differences were found between linear measurements performed $24 \mathrm{~h}$ after polymerization and after 30 days of water storage in all subgroups (ROUNDED/NOF, ROUNDED/WOF, ROUNDED/ROF) ( $\mathrm{P}<.01)$ (Table 6).

Table 4. Mean, standard deviation and $P$ values of data $(\mathrm{mm})$ obtained from linear measurements of distances between 4 references points $(A, B, C$, and $D)$ in Uium palatal vault rounded groups $24 \mathrm{~h}$ after polymerization and after 30 days of water storage $\left.{ }^{*}=P<.05\right)$.

\begin{tabular}{|c|c|c|c|c|c|c|c|c|c|c|}
\hline \multirow{2}{*}{$\begin{array}{l}\text { Groups } \\
\text { Distances }\end{array}$} & \multirow[b]{2}{*}{$\begin{array}{l}\text { Time of linear } \\
\text { measurement }\end{array}$} & \multicolumn{3}{|c|}{ U/NOF } & \multicolumn{3}{|c|}{ U/WOF } & \multicolumn{3}{|c|}{ U/ROF } \\
\hline & & Mean & St. Dev. & $P$ & Mean & St. Dev. & $\mathrm{P}$ & Mean & St. Dev. & $P$ \\
\hline \multirow{2}{*}{$A-B$} & 24 h (a.p.) & 46.310 & 0.367 & $<0.01^{*}$ & 46.570 & 0.287 & $<0.01 *$ & 46.380 & 0.297 & $<0.01 *$ \\
\hline & 30 days (w.s.) & 46.970 & 0.654 & $<0.01^{*}$ & 46.900 & 0.254 & $<0.01^{*}$ & 46.910 & 0.191 & $<0.01 *$ \\
\hline \multirow{2}{*}{$A-C$} & 24 h (a.p.) & 44.130 & 0.216 & $<0.01^{*}$ & 44.760 & 0.570 & $<0.01^{*}$ & 44.270 & 0.221 & $<0.01 *$ \\
\hline & 30 days (w.s.) & 44.830 & 0.538 & $<0.01 *$ & 45.120 & 0.571 & $<0.01^{*}$ & 44.810 & 0.443 & $<0.01 *$ \\
\hline \multirow{2}{*}{$A-D$} & 24 h (a.p.) & 46.060 & 0.320 & $<0.01^{*}$ & 45.290 & 0.601 & $<0.01^{*}$ & 45.140 & 0.165 & $<0.01 *$ \\
\hline & 30 days (w.s.) & 46.610 & 0.526 & $<0.01^{*}$ & 45.570 & 0.552 & $<0.01 *$ & 45.600 & 0.302 & $<0.01 *$ \\
\hline \multirow{2}{*}{$B-C$} & 24 h (a.p.) & 43.860 & 0.284 & $<0.01 *$ & 44.120 & 0.215 & $<0.01^{*}$ & 43.930 & 0.206 & $<0.01 *$ \\
\hline & 30 days (w.s.) & 44.530 & 0.263 & $<0.01^{*}$ & 44.350 & 0.212 & $<0.01^{*}$ & 44.350 & 0.360 & $<0.01 *$ \\
\hline
\end{tabular}

Table 5. Mean, standard deviation and $P$ values of data $(\mathrm{mm})$ obtained from linear measurements of distances between 4 references points $(A, B, C$, and $D)$ in $V$ palatal vault rounded groups $24 \mathrm{~h}$ after polymerization and after 30 days of water storage $\left.~_{*}^{*}=\mathrm{P}<.05\right)$.

\begin{tabular}{|c|c|c|c|c|c|c|c|c|c|c|}
\hline Groups & & & V/NOF & & & V/WOF & & & ROF & \\
\hline Distances & $\begin{array}{l}\text { Time of linear } \\
\text { measurement }\end{array}$ & Mean & St. Dev & $P$ & Mean & St. Dev & $P$ & Mean & St. Dev & $\mathrm{P}$ \\
\hline \multirow{2}{*}{$A-B$} & 24 h (a.p.) & 50.160 & 0.184 & \multirow{2}{*}{$<0.01 *$} & 50.190 & 0.233 & \multirow{2}{*}{$<0.01 *$} & 50.110 & 0.292 & \multirow{2}{*}{$<0.01 *$} \\
\hline & 30 days (w.s.) & 50.470 & 0.211 & & 50.600 & 0.245 & & 50.460 & 0.310 & \\
\hline \multirow{2}{*}{$A-C$} & 24 h (a.p.) & 50.870 & 0.149 & \multirow{2}{*}{$<0.01 *$} & 50.770 & 0.330 & \multirow{2}{*}{$<0.01 *$} & 50.680 & 0.308 & \multirow{2}{*}{$<0.01 *$} \\
\hline & 30 days (w.s.) & 51.080 & 0.114 & & 51.130 & 0.231 & & 51.060 & 0.135 & \\
\hline \multirow{2}{*}{$A-D$} & $24 \mathrm{~h}$ (a.p.) & 48.970 & 0.236 & \multirow{2}{*}{$<0.01 *$} & 48.930 & 0.183 & \multirow{2}{*}{$<0.01 *$} & 49.060 & 0.107 & \multirow{2}{*}{$<0.01 *$} \\
\hline & 30 days (w.s.) & 49.270 & 0.164 & & 49.270 & 0.125 & & 49.330 & 0.125 & \\
\hline \multirow[b]{2}{*}{$B-C$} & 24 h (a.p.) & 48.980 & 0.193 & \multirow{2}{*}{$<0.01 *$} & 48.890 & 0.335 & \multirow[b]{2}{*}{$<0.01 *$} & 48.940 & 0.207 & \multirow[b]{2}{*}{$<0.01 *$} \\
\hline & 30 days (w.s.) & 49.280 & 0.123 & & 43.298 & 0.273 & & 49.360 & 0.241 & \\
\hline
\end{tabular}

Table 6. Mean, standard deviation and $P$ values of data $(\mathrm{mm})$ obtained from linear measurements of distances between 4 references points $(\mathrm{A}, \mathrm{B}, \mathrm{C}$, and $\mathrm{D})$ in rounded palatal vault rounded groups $24 \mathrm{~h}$ after polymerization and after 30 days of water storage.

\begin{tabular}{|c|c|c|c|c|c|c|c|c|c|c|}
\hline \multirow{2}{*}{$\begin{array}{l}\text { Groups } \\
\text { Distances }\end{array}$} & \multirow[b]{2}{*}{$\begin{array}{l}\text { Time of linear } \\
\text { measurement }\end{array}$} & \multicolumn{3}{|c|}{ ROUNDED/NOF } & \multicolumn{3}{|c|}{ ROUNDED/WOF } & \multicolumn{3}{|c|}{ ROUNDED/ROF } \\
\hline & & Mean & St. Dev & $\mathrm{P}$ & Mean & St. Dev & $\mathrm{P}$ & Mean & St. Dev & $\mathrm{P}$ \\
\hline \multirow{2}{*}{$A-B$} & 24 h (a.p.) & 46,890 & 0.472 & \multirow{2}{*}{$<0.01 *$} & 47,350 & 0.246 & \multirow{2}{*}{$<0.01 *$} & 47,570 & 0.245 & \multirow{2}{*}{$<0.01 *$} \\
\hline & 30 days (w.s.) & 47,430 & 0.377 & & 47,840 & 0.250 & & 47,470 & 0.157 & \\
\hline \multirow{2}{*}{$A-C$} & $24 \mathrm{~h}$ (a.p.) & 46,030 & 0.316 & \multirow{2}{*}{$<0.01 *$} & 46,320 & 0.169 & \multirow{2}{*}{$<0.01 *$} & 46,610 & 0.269 & \multirow{2}{*}{$<0.01 *$} \\
\hline & 30 days (w.s.) & 46,550 & 0.317 & & 46,820 & 0.169 & & 46,890 & 0.152 & \\
\hline \multirow{2}{*}{$A-D$} & 24 h (a.p.) & 45,760 & 0.366 & \multirow{2}{*}{$<0.01 *$} & 46,000 & 0.183 & \multirow{2}{*}{$<0.01 *$} & 46,100 & 0.267 & \multirow{2}{*}{$<0.01 *$} \\
\hline & 30 days (w.s.) & 46,230 & 0.267 & & 46,430 & 0.183 & & 46,430 & 0.283 & \\
\hline \multirow{2}{*}{$B-C$} & 24 h (a.p.) & 53,940 & 0.232 & \multirow{2}{*}{$<0.01 *$} & 54,250 & 0.190 & \multirow{2}{*}{$<0.01 *$} & 54,270 & 0.275 & \multirow{2}{*}{$<0.01 *$} \\
\hline & 30 days (w.s.) & 54,390 & 0.218 & & 54,630 & 0.231 & & 54,660 & 0.217 & \\
\hline
\end{tabular}

$\left(^{*}=p<0.05\right)$ 


\section{DISCUSSION}

Polymerization shrinkage and water sorption, in other words, the parameters affecting the dimensional stability of acrylic resins denture base materials have been the subject of numerous studies. ${ }^{2-7}$ Thickness of the denture, thermal expansion and contraction of the acrylic resin and the gypsum, structural variations between polymer and monomer systems, polymer/monomer ratios, packing pressure, polymerization shrinkage, investments, separators, internal stresses, water sorption and desorption and water content of the gypsum were reported as other parameters influencing the dimensional stability of an acrylic resin denture. ${ }^{2-10}$ Two methods were mostly used for the determination of dimensional alterations in acrylic resin denture base materials: Determination of the present closeness (adaptation) of acrylic resin base to the definitive stone cast surface, ${ }^{6,7,18}$ and distance measurement between determined reference points on an acrylic resin denture base. ${ }^{3,5,8}$ Both methods were used in the present study to provide reliable results. This method provided real-time direct dimensional changes of the denture base resin during the fabrication process, as reported by Kawara et al. ${ }^{22}$

Heat polymerized acrylic resin denture base material and conventional flasking technique were used in the present study due to the ease of processing and to minimize the complications faced during the impregnation of glass fibers into the acrylic resin bulk.

Metal wires, grids and various types of metal plates were used previously to strengthen the acrylic resin dentures base materials. $6,15,16$ However, despite of their satisfactory strenghtening effect, these reinforcing materials were too far from providing even minimal esthetics.6,15,16 The introduction of reinforcing fibers of various types and forms in the field of prosthetic dentistry greatly solved this problem. ${ }^{11-14}$ It was reported in previous studies $^{16,18-21}$ that glass fibers provided satisfactory impregnation with PMMA, as well as good esthetics. Thus, in the present study, wowen and roving type glass fibers were used to provide successful incorporation of fibers with acrylic resin bulk. A second reason for preferring glass fibers was their rare shifting occurrence during flasking of acrylic resin, due to their density. ${ }^{17,23}$

The results of the present study demonstrated that palatal vault shape and fiber impregnation into the acrylic resin bases did not affect the magnitude of interfacial gaps between the bases and the stone cast surfaces, after 30 days of water storage. This finding is in correlation with the statements of Polat et al $^{19}$ who reported that the dimensional stability of acrylic resin denture base material used was not affected with fiber reinforcement. In the present study it was shown that water storage of 30 days significantly affected the linear distances between references points marked on acrylic resin bases in all experimental groups. Contrary to these findings, Cal et al ${ }^{18}$ reported that the unreinforced group showed the highest dimensional change compared to fiber reinforced groups.

The effect of the shape of residual mandibular crest and effects other fiber types on the dimensional stability of different acrylic resin denture base materials are to be investigated in future studies.

\section{CONCLUSION}

1. The interfacial distance between the acrylic resin and the relevant stone cast is independent of palatal vault shape and from fiber reinforcement.

2. Water storage of 30 days significantly affected the linear dimensional stability between references points marked on acrylic resin bases in all experimental groups.

\section{REFERENCES}

1. Brown LR, Flavin C, French H. A new economy for a new century. In: State of the world 1999. New York: W.W. Norton \& Company $1999 ; 5-7$

2. Wolfaardt J, Cleaton-Jones P, Fatti P. The influence of processing variables on dimensional changes of heat-cured poly(methyl methacrylate). J Prosthet Dent 1986;55:518525.

3. Anderson GC, Schulte JK, Arnold TG. Dimensional stability of injection and conventional processing of denture base resin. J Prosthet Dent 1988;60:394-398.

4. Phillips RW. Skinner's Science of Dental Materials. 1991; $9^{\text {th }}$ Ed. W.B. Saunders Co, Philadelphia, 177-213.

5. Huggett R, Zissis A, Harrison A, Dennis A. Dimensional accuracy and stability of acrylic resin denture bases. J Prosthet Dent 1992;68:634-640.

6. Dixon DL, Breeding LC, Ekstrand KG. Linear dimensional variability of three denture base resins after processing and in water storage. J Prosthet Dent 1992;68:196-200. 
7. Salim S, Sadamori S, Hamada T. The dimensional accuracy of rectangular acrylic resin specimens cured by three denture base processing methods. J Prosthet Dent 1992;67:879881.

8. Turck MD, Lang BR, Wilcox DE, Meiers JC. Direct measurement of dimensional accuracy with three denture-processing techniques. Int J Prosthodont 1992;5:367-372.

9. Pronych GJ, Sutow EJ, Sykora O. Dimensional stability and dehydration of a thermoplastic polycarbonate based and two PMMA based denture resins. J Oral Rehabil 2003;30:1157-1161.

10. Monfrin SB, Notaro V, Gassino G, Perotti R, Bassi F. Dimensional contour stability of acrylic resin bases for complete dentures before and after water sorption. Int J Prosthodont 2005; 18 480-482.

11. Clarke DA, Ladizesky NH, Chow TW. Acrylic resins reinforced with highly drawn linear polyethylene woven fibres. Part I. Construction of upper denture bases. Aust Dent J 1992;37:394-399.

12. Ladizesky NH, Cheng YY, Chow TW, Ward IM. Acrylic resin reinforced with chopped high performance polyethylene fiber. Properties and denture construction. Dent Mater 1993;9:128-135.

13. Ladizesky NH, Chow TW. Reinforcement of complete denture bases with continuous high performance polyethylene fibers. J Prosthet Dent 1992;68:934-939.

14. Ladizesky NH, Pang MK, Chow TW, Ward IM. Acrylic resins reinforced with woven highly drawn linear polyethylene fibres. Part III. Mechanical properties and further aspects of denture construction. Aust Dent J 1993;38:28-38.

15. Vallittu PK. Acrylic resin-fiber composite. Part II: The effect of polymerization shrinkage of polymethyl methacrylate applied to fiber roving on transverse strength. $J$ Prosthet Dent 1994;71:613-617.

16. Vallittu PK. Dimensional accuracy and stability of polymethyl methacrylate reinforced with metal wire or with continuous glass fiber. J Prosthet Dent 1996;75:617-621.

17. Vallittu PK. Some aspects of the tensile strength of unidirectional glass fiber-polymethyl methacrylate composite used in dentures. J Oral Rehabil 1998;25:100-105.

18. Cal NE, Hersek N, Sahin E. Water sorption and dimensional changes of denture base polymer reinforced with glass fibers in continuous unidirectional and woven form. Int $J$ Prosthodont 2000;13;487-493.

19. Polat TN, Karacaer O, Tezvergil A, Lassila LV, Vallittu PK. Water sorption, solubility and dimensional changes of denture base polymers reinforced with short glass fibers. J Biomater Appl 2003;17:321-335.
20. Karacaer O, Polat TN, Tezvergil A, Lassila LV, Vallittu PK. The effect of length and concentration of glass fibers on the mechanical properties of an injection- and a compressionmolded denture base polymer. J Prosthet Dent 2003;90:385393.

21. Ozturk AN, Inan 0, Yondem I. Dimensional changes and water sorption of two acrylic polymer reinforced with glass fibres. Eur J Prosthodont Restor Dent 2003;11:129-132.

22. Kawara M, Komiyama O, Kimoto S, Kobayashi N, Kobayashi K,Nemoto K. Distortion behavior of heat-activated acrylic denture-base resin in conventional and long, low temperature processing methods. J Dent Res 1998;77:1446-1453.

23. Vallittu PK. The effect of glass fiber reinforcement on the fracture resistance of a provisional fixed partial denture. $J$ Prosthet Dent 1998;79:125-130. 